\title{
Co-pyrolysis behavior of xylan and polyvinyl chloride (PVC) plastic
}

3 Wanli Wang ${ }^{a}$, Kai Sun ${ }^{a}$, Mujahid Ali ${ }^{a}$, Xiaoji Liu ${ }^{b}$, Qunxing Huang ${ }^{a, *}$

$4{ }^{a}$ State Key Laboratory of Clean Energy Utilization, Zhejiang University, Hangzhou, 310027,

$5 \quad$ People's Republic of China

$6{ }^{b}$ China National Environmental Protection Group, Beijing 100082, People's Republic of China

7 * Corresponding Author Telephone: +86-571-87952834. Fax: +86-571-87952438. E-mail:

8 hqx@zju.edu.cn

9 Supporting Information

\section{Table of Contents}

11 Page S2-S5: Main compositions of xylan and PVC from Py-GC-MS results (based on area \%).

12 (Table S1) 
13 Table S1. Main compositions of pyrolysis oils (based on area \%).

\begin{tabular}{|c|c|c|}
\hline Identified compound & $\begin{array}{l}\text { Chemical } \\
\text { formula }\end{array}$ & Area $\%$ \\
\hline \multicolumn{3}{|l|}{ PVC } \\
\hline Benzene & $\mathrm{C}_{6} \mathrm{H}_{6}$ & 58.48 \\
\hline 1H-Indene, 1-methylene- & $\mathrm{C}_{10} \mathrm{H}_{8}$ & 11.04 \\
\hline Naphthalene, 2-methyl- & $\mathrm{C}_{11} \mathrm{H}_{10}$ & 5.90 \\
\hline Cyclobutene, 2-propenylidene- & $\mathrm{C}_{7} \mathrm{H}_{8}$ & 5.18 \\
\hline Phenanthrene & $\mathrm{C}_{14} \mathrm{H}_{10}$ & 4.69 \\
\hline Biphenyl & $\mathrm{C}_{12} \mathrm{H}_{10}$ & 2.94 \\
\hline Indene & $\mathrm{C}_{10} \mathrm{H}_{8}$ & 2.93 \\
\hline Fluorene & $\mathrm{C}_{10} \mathrm{H}_{8}$ & 2.30 \\
\hline Styrene & $\mathrm{C}_{10} \mathrm{H}_{8}$ & 2.17 \\
\hline 1,4-Ethenonaphthalene, 1,4-dihydro- & $\mathrm{C}_{10} \mathrm{H}_{8}$ & 1.64 \\
\hline 1,1'-Biphenyl, 4-methyl- & $\mathrm{C}_{10} \mathrm{H}_{8}$ & 1.42 \\
\hline p-Xylene & $\mathrm{C}_{10} \mathrm{H}_{8}$ & 1.28 \\
\hline \multicolumn{3}{|l|}{80 wt $\%$ PVC+20 wt\%Xylan } \\
\hline Benzene & $\mathrm{C}_{6} \mathrm{H}_{6}$ & 25.59 \\
\hline Naphthalene, 1-methyl- & $\mathrm{C}_{11} \mathrm{H}_{10}$ & 9.93 \\
\hline Furfural & $\mathrm{C}_{5} \mathrm{H}_{4} \mathrm{O}_{2}$ & 8.67 \\
\hline 1H-Indene, 1-methylene- & $\mathrm{C}_{10} \mathrm{H}_{8}$ & 6.66 \\
\hline Toluene & $\mathrm{C}_{7} \mathrm{H}_{8}$ & 6.42 \\
\hline dl-2-Ethylhexyl chloroformate & $\mathrm{C}_{9} \mathrm{H}_{17} \mathrm{O}_{2}$ & 5.93 \\
\hline Phenanthrene & $\mathrm{C}_{14} \mathrm{H}_{10}$ & 4.57 \\
\hline 2,6,10,14,18,22-Tetracosahexaene,2,6,10,15,19,23-hexamethyl-, (all-E)- & $\mathrm{C}_{30} \mathrm{H}_{50}$ & 4.14 \\
\hline
\end{tabular}


Styrene

$\mathrm{C}_{8} \mathrm{H}_{8}$

1,4-Methanonaphthalen-9-one,1,2,3,4-tetrahydro-

$\mathrm{C}_{11} \mathrm{H}_{10} \mathrm{O} \quad 3.66$

Benzene, 1-ethynyl-4-methyl-

$\mathrm{C}_{9} \mathrm{H}_{8}$

2.38

1H-Phenalene

$\mathrm{C}_{13} \mathrm{H}_{10}$

2.37

Naphthalene, 2-ethenyl-

$$
\mathrm{C}_{12} \mathrm{H}_{10}
$$

1,1'-Biphenyl, 4-methyl-

$\mathrm{C}_{13} \mathrm{H}_{12}$

2.20

Biphenyl

$\mathrm{C}_{12} \mathrm{H}_{10}$

2.06

o-Xylene

$\mathrm{C}_{8} \mathrm{H}_{10}$

8,9-Dihydrocyclopenta[def]phenanthrene

$\mathrm{C}_{15} \mathrm{H}_{12}$

1.72

Acetic acid

$\mathrm{C}_{2} \mathrm{H}_{4} \mathrm{O}_{2}$

1.72

1,1'-Biphenyl, 4-methyl-

$\mathrm{C}_{13} \mathrm{H}_{12}$

5H-Dibenzo[a,d]cycloheptene, 5-methylene-

$\mathrm{C}_{16} \mathrm{H}_{12}$

9H-Fluorene, 4-methyl-

$\mathrm{C}_{14} \mathrm{H}_{12}$

1.30

\section{$60 \mathrm{wt} \% \mathrm{PVC}+40$ wt\%Xylan}

3-Furaldehyde

$\mathrm{C}_{5} \mathrm{H}_{4} \mathrm{O}_{2}$

31.23

Benzene

$\mathrm{C}_{6} \mathrm{H}_{6}$

19.63

Naphthalene, 1-methyl-

$\mathrm{C}_{11} \mathrm{H}_{10}$

6.41

Toluene

$\mathrm{C}_{7} \mathrm{H}_{8}$

6.32

n-Hexadecanoic acid

$\mathrm{C}_{16} \mathrm{H}_{32} \mathrm{O}_{2} \quad 5.16$

1H-Indene, 1-methylene-

$\mathrm{C}_{10} \mathrm{H}_{8}$

3.88

2-Butanone, 3,4-epoxy-3-ethyl-

$\mathrm{C}_{6} \mathrm{H}_{10} \mathrm{O}_{2} \quad 2.71$

Acetic acid

$\mathrm{C}_{2} \mathrm{H}_{4} \mathrm{O}_{2}$

2.67

Butane, 2-cyclopropyl-

$\mathrm{C}_{7} \mathrm{H}_{14}$

2.47

2-Furancarboxaldehyde, 5-(hydroxymethyl)-

$\mathrm{C}_{6} \mathrm{H}_{6} \mathrm{O}_{3}$

2.40

2,6-Dimethyl-3,7,9-trioxabicyclo[4.2.1]nonane

$\mathrm{C}_{8} \mathrm{H}_{14} \mathrm{O}_{3}$

2.30

Phenanthrene

$\mathrm{C}_{14} \mathrm{H}_{10}$

2.32 
2-Cyclopenten-1-one, 2-hydroxy-

$\mathrm{C}_{5} \mathrm{H}_{6} \mathrm{O}_{2} \quad 2.26$

1,4-Methanonaphthalen-9-one,1,2,3,4-tetrahydro-

$\mathrm{C}_{11} \mathrm{H}_{10} \mathrm{O}$

2.19

(SS)- or (RR)-4-methyl-2,3-pentanediol

$\mathrm{C}_{16} \mathrm{H}_{14} \mathrm{O}_{2}$

1.92

1H-Phenalene

$\mathrm{C}_{13} \mathrm{H}_{10}$

1.72

Pentanedioic acid, 3-methyl-, dibutyl ester

$\mathrm{C}_{14} \mathrm{H}_{26} \mathrm{O}_{4}$

1.57

Styrene

$\mathrm{C}_{8} \mathrm{H}_{8}$

1.52

1,1'-Biphenyl, 4-methyl-

$\mathrm{C}_{13} \mathrm{H}_{12}$

1.46

40 wt\%PVC+60 wt\%Xylan

3-Furaldehyde

$\mathrm{C}_{5} \mathrm{H}_{4} \mathrm{O}_{2}$

45.50

Benzene

$\mathrm{C}_{6} \mathrm{H}_{6}$

14.83

Naphthalene, 1-methyl-

$\mathrm{C}_{11} \mathrm{H}_{10}$

4.61

Toluene

$\mathrm{C}_{7} \mathrm{H}_{8}$

4.33

2-Butanone, 3,4-epoxy-3-ethyl-

$\mathrm{C}_{6} \mathrm{H}_{10} \mathrm{O}_{2}$

4.13

meso-5,6-Decanediol

$\mathrm{C}_{10} \mathrm{H}_{22} \mathrm{O}_{2}$

3.86

Butane, 2-cyclopropyl-

$\mathrm{C}_{7} \mathrm{H}_{14}$

3.69

1H-Indene, 1-methylene-

$\mathrm{C}_{10} \mathrm{H}_{8}$

3.11

2-Furancarboxaldehyde, 5-(hydroxymethyl)-

$\mathrm{C}_{6} \mathrm{H}_{6} \mathrm{O}_{3}$

2.88

4-Tetradecanol

$\mathrm{C}_{14} \mathrm{H}_{30} \mathrm{O}_{1}$

2.43

2-Cyclopenten-1-one, 2-hydroxy-

$\mathrm{C}_{5} \mathrm{H}_{6} \mathrm{O}_{2}$

2.34

Acetic acid

$\mathrm{C}_{2} \mathrm{H}_{4} \mathrm{O}_{2}$

1.84

Phenanthrene

$\mathrm{C}_{14} \mathrm{H}_{10}$

1.72

1H-Phenalene

$\mathrm{C}_{13} \mathrm{H}_{10}$

1.70

Sucrose

$\mathrm{C}_{12} \mathrm{H}_{22} \mathrm{O}_{11} \quad 1.62$

Styrene

$\mathrm{C}_{8} \mathrm{H}_{8}$

1.40

20 wt\%PVC +80 wt\%Xylan

3-Furaldehyde

$\mathrm{C}_{5} \mathrm{H}_{4} \mathrm{O}_{2}$

70.37 
2-Furancarboxaldehyde, 5-(hydroxymethyl)-
$\mathrm{C}_{6} \mathrm{H}_{6} \mathrm{O}_{3}$
8.11
$\mathrm{C}_{10} \mathrm{H}_{20} \mathrm{O}_{1} \quad 6.99$
$\mathrm{C}_{4} \mathrm{H}_{6} \mathrm{O}_{1} \quad 5.06$
$\mathrm{C}_{6} \mathrm{H}_{6}$
4.33
$\mathrm{C}_{5} \mathrm{H}_{6} \mathrm{O}_{2}$
3.31
$\mathrm{C}_{6} \mathrm{H}_{12} \mathrm{O}_{3}$
1.83

3,6-Dimethyl-4-octanone

(E)-1,3-Butadien-1-ol

Benzene

2-Furanmethanol

$[1,4,7]$ Trioxonane

\section{Xylan}

3-Furaldehyde

$\mathrm{C}_{5} \mathrm{H}_{4} \mathrm{O}_{2} \quad 38.73$

Sucrose

$\mathrm{C}_{12} \mathrm{H}_{22} \mathrm{O}_{11} \quad 38.39$

(SS)- or (RR)-4-methyl-2,3-pentanediol

$\mathrm{C}_{6} \mathrm{H}_{14} \mathrm{O}_{2} \quad 5.34$

2-Furancarboxaldehyde, 5-(hydroxymethyl)-

$\mathrm{C}_{6} \mathrm{H}_{6} \mathrm{O}_{3} \quad 4.52$

2-Butanone, 3,4-epoxy-3-ethyl-

$\mathrm{C}_{6} \mathrm{H}_{10} \mathrm{O}_{2} \quad 4.18$

2-Furanmethanol

$\mathrm{C}_{5} \mathrm{H}_{6} \mathrm{O}_{2} \quad 2.62$

1-Ethylcyclopropanol

$\mathrm{C}_{5} \mathrm{H}_{10} \mathrm{O}_{1}$

2.15

Butane, 2-cyclopropyl-

$\mathrm{C}_{7} \mathrm{H}_{14}$

1.55

Butanedial

$\mathrm{C}_{4} \mathrm{H}_{6} \mathrm{O}_{2}$

1.33

d-Arabinal

$\mathrm{C}_{5} \mathrm{H}_{8} \mathrm{O}_{3}$

1.20 